\title{
Werbekommunikation: Drei sprachliche Strategien, um das Werbeprodukt zu verbergen.
}

\author{
Federica Ricci Garotti (Trento)
}

\begin{abstract}
The language of advertising uses many techniques for persuasion: focusing on emotions and positive words while hiding negative or important information. Based on the idea that one of the most used strategies of advertising is the absence of the product to sell, the article will focus on the advertising ability to persuade large masses of people without showing the product itself.
\end{abstract}

\section{$1 \quad$ Einleitung}

Im folgenden Beitrag werden drei von zahlreichen Strategien beleuchtet, die in der Werbebotschaft mittels Mehrdeutigkeit, Sprachspiele und Inszenierung die vielfältigen kommunikativen Ausdrucksmöglichkeiten der Werbung nutzen, um das Produkt zu verstecken. Das Verbergen des Produkts ist keine Paradoxie, sondern ein Grundprinzip moderner und postmoderner Werbung, das viele Forscher/Forscherinnen in ihren Studien hervorgehoben haben. 2008 hatte Stöckl die Tendenz, das Produkt und seine Eigenschaften in den Hintergrund treten zu lassen, als eine der wichtigsten Eigenschaften der Werbung nach den 50er Jahren identifiziert (Stöckl 2008: 171), um die heute zunehmende „Langeweile, Aversion und Vermeidungsstrategien der Empfänger" (Knoblauch/Raab 2002: 139) zu überwinden. Einerseits braucht die Werbung als funktional-persuasive Kommunikation raffinierte Techniken und ästhetische Stilmittel (Musik, Bilder, Implikationen), die stets zu einer der für das Auge und das Ohr angenehmsten Formen der modernen Alltagskultur zählen; auf der anderen Seite bewirken die allzu geschickten, pfiffigen Kommunikations- und Darstellungsstrategien, die gern auf ihren informativen Zweck verzichten, eine zweifelnde Reaktion der Empfänger, die im Laufe der Jahre immer mehr werbeskeptisch und werberesistent geworden sind. Je impliziter und unglaubwürdiger die Werbeinszenierung sich gestaltet, desto ungläubiger und kritischer reagieren die Empfänger/Empfängerinnen. Die von Janich aufgezeigten Möglichkeiten der Werbung, mehrdeutige Assoziationen und implizite Bedeutungen zu erwecken (und zu erkennen), werden heutzutage von den Werbegestaltern vorsichtig verwendet. Homonymie, Polysemie, Synonymie, Antonyme, Sprachspiele (Janich 2003: 68-69) dienen als Zeichen der Realisierung eines größeren Gesamtbilds zur Vermittlung von Emotionen und ästhetischem Vergnügen, die weit über die von der Ambiguität und dem spielerischen Umgang mit der Sprache ermittelte Neugier hinaus gehen. Deshalb bedient sich die Werbung raffinierterer und 
impliziter Inszenierungsstrategien, die die Rolle der Werbesender als Verkäufer verstecken und somit auf die Darstellung des Produkts als Fokus des Werbetexts verzichten.

Um die Strategien des Verbergens zu analysieren, hatte ich ein Korpus von 30 deutschen Anzeigen zur Verfügung. Die Anzeigen wurden seit Januar bis Mai 2017 von mir aus den deutschen Zeitschriften „Der Spiegel“, „Focus“ und „Stern“ eingesammelt. Diese Zeitschriften wurden nach dem Kriterium der Popularität selektiert: Sie erscheinen in der Liste der 2017 in Deutschland auflagenstärksten und meistverkauften Zeitschriften, die an ein allgemeines Publikum gerichtet sind (cf. Schröder 2017). Die ausgewählten Anzeigen treten aufgrund der Häufigkeit in mein Korpus ein, mit der sie in den erwähnten Zeitschriften erscheinen. Eine Ausnahme stellt die Werbung von SEB dar (Abbildung 5): Das Plakat wurde von mir persönlich im August 2014 in Graz photographiert.

Bei der Mehrheit der Anzeigen (25 davon) habe ich Strategien des Produkt-Verbergens festgestellt. Im Folgenden gehe ich auf die drei Strategien ein, die die meisten Anzeigen aufzeigten: Lösung eines Problems, Themawechsel, scheinbare Popularisierung.

Hierzu werden nur Beispiele von - nicht alle -Anzeigen wiedergegeben, in denen die erwähnten Strategien realisiert sind. Da das Ziel meiner Studie keine quantitative Erhebung, sondern eine qualitative Analyse kommunikativer Persuasionsstrategien ist, wird die Gesamtheit der gesammelten und analysierten Anzeigen als Appendix 1 tabellarisch gezeigt.

Nach der kurzen Einleitung werden im Beitrag drei Strategien des Produktverbergens illustriert, die in den meisten Korpusanzeigen auffielen. Jeder Strategie wird ein Abschnitt gewidmet, in dem die jeweiligen Anzeigen als Beispiele angeboten werden: Im zweiten Abschnitt wird die Strategie der Problemlösung gezeigt, die durch Sprechakte benutzt wird, deren Gesamtillokution die Voraussetzung eines Problems der Empfänger/Empfängerinnen ist, das die Werbung lösen kann. Der Themawechsel ist die zweite betrachtete Strategie des Verbergens, die im dritten Abschnitt analysiert wird: Die betreffenden Anzeigen realisieren solche Strategie vor allem mittels Stereotypen und besonderer Kombination von Wörtern. Die dritte und letzte Strategie wird im vierten Abschnitt durch die Analyse von Fachwörtern erklärt, die die Illusion einer Popularisierung durch die Werbung, nämlich die Verbreitung von Fachkenntnissen, aufbauen. Im letzten Abschnitt, Fazit und Schlusswort, geht man auf das beschriebene Phänomen des Produktverbergens zusammenfassend ein, indem man es als notwendigen Bestandteil moderner Werbung bezeichnet, der nicht ohne die (unbeabsichtigte?) Teilnahme der Empfänger/Empfängerinnen vorkommt: Einig ist sich die Forschung darin, dass Empfänger/Empfängerinnen nicht mehr die naive und unbewusste Rolle des Simplicissimus spielen, der sich vollständig beeinflussen lässt, sondern sie garantieren eher eine aktive und notwendige Teilnahme im kommunikativen Pakt zwischen Werbeproduzenten und Publikum. Eigentlich rechnet man in der Werbekommunikation mit der von Weinrich als ,Schuld der Rezipienten“ bezeichneten Mitwirkung (Weinrich 2000: 5), weil im Mittelpunkt der Werbekommunikation nicht die Lüge steht, sondern eher das Bewusstsein, etwas zu inszenieren, anstatt die Realität widerzuspiegeln. 


\section{$2 \quad$ Werbung als Problemlösung}

Im Folgenden sind Beispiele einiger Anzeigen zu sehen, die auf eine unausgesprochene Frage bzw. ein nicht genanntes Problem der Empfänger/Empfängerinnen Bezug nehmen und eine Antwort darauf geben (Abbildung 1 und 2). Natürlich impliziert die angeratene Lösung die Benutzung des geworbenen Produkts. Jedoch steht das Problem, und nicht das Produkt, im Mittelpunkt der Werbekampagne, als ob die Werbung den Empfängern/Empfängerinnen endlich die Wörter zur Verfügung stelle, um das Problem darzustellen. Wie im Roman Schattenmund. Die Wörter, um es zu sagen von Marie Cardinal (1983), in der die Protagonistin fest davon überzeugt ist, durch die Verbalisierung ihrer ungelösten Konflikte ihr Leben von Grund auf zu verändern und zu gesunden, übernimmt Werbung die Rolle des Auslösers und Heilmittels mittels der expliziten Darstellung des Problems, die verbal oder verbal und bildlich vorkommen kann, wie die hier wiedergegebenen Anzeigen zeigen: verbale Thematisierung: „die einseitige Ernährung“; verbale und bildliche Darstellung: „trockenes, widerspenstiges Haar?“ plus das zu verbrennende Bild einer Dame mit kräuseligem Haar.

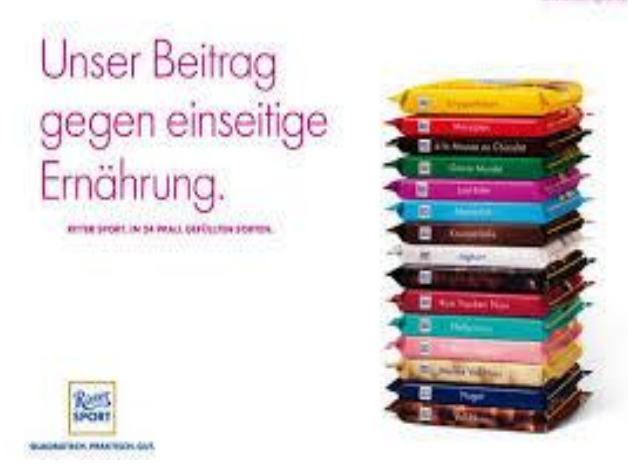

Abbildung 1

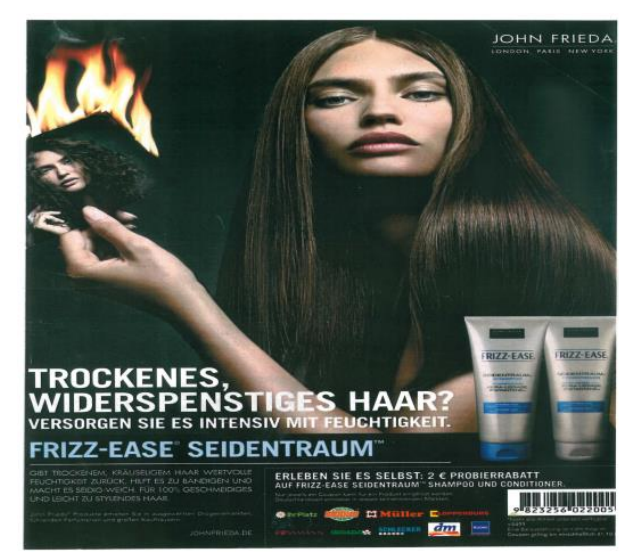

Abbildung 2

Die Thematisierung des Problems sieht vor, dass für die sprachliche Realisierung meistens auf negative Formen Zugriff genommen wird, auf die in der Regel in der Werbung gerne verzichtet wird, um die positive, schillernde Lösung des Problems kontrastiv hervorheben zu können. Die negative Darstellung der Realität vor der und ohne die Verwendung des beworbenen Produkts wird mittels Präfixe und Präpositionen realisiert (gegen, wider, und). Weitere Realisierungsformen sind, wie im Fall von Frizz-Ease, die rhetorische Frage oder Wörter für negative Emotionen bzw. Gefühle (Schmerz, Wunde). 
In einem solchen Werbedesign bedienen sich die Werbeproduzenten auch expliziter Imperativformen, die eigentlich zu den ältesten und längst veralteten Werbestrategien gehören, weil das Gefühl einer direkten Aufforderung zu explizit auf die Empfänger/Empfängerinnen einwirkt. In der folgenden Anzeige (Abbildung 3) von Nivea wird die direkte Mitwirkung der Empfängerin gegen ihr eigenes Problem durch die Imperativform verstärkt: „Entdecken Sie das Geheimrezept der Haut gegen Falten!“' In diesem Fall wird das Problem sozusagen entpersonalisiert: Problematisch ist nicht die angesprochene Empfängerin, sondern ihre Haut, die eine Lösung braucht. Da die Empfängerin in enger Beziehung mit ihrer Haut steht, kann sie keine Lösung finden und so wirkt die Werbeaufforderung eher als Aufmunterung als Befehl.

Die Werbeproduzenten können sich in den gezeigten Beispielen diese Explizitheit leisten, weil sie hier nicht als Verkäufer/Händler/Werbewirtschaftler handeln, sondern als Retter/Unterstützer/Helfer. Die Hilfsbereitschaft, das Verständnis für die Sorgen der Anderen und ihr Entgegenkommen mittels Vorschläge und Antworten, die nicht angefragt wurden, kennzeichnen die Werbekampagne und das realisierte sprachliche Handeln.

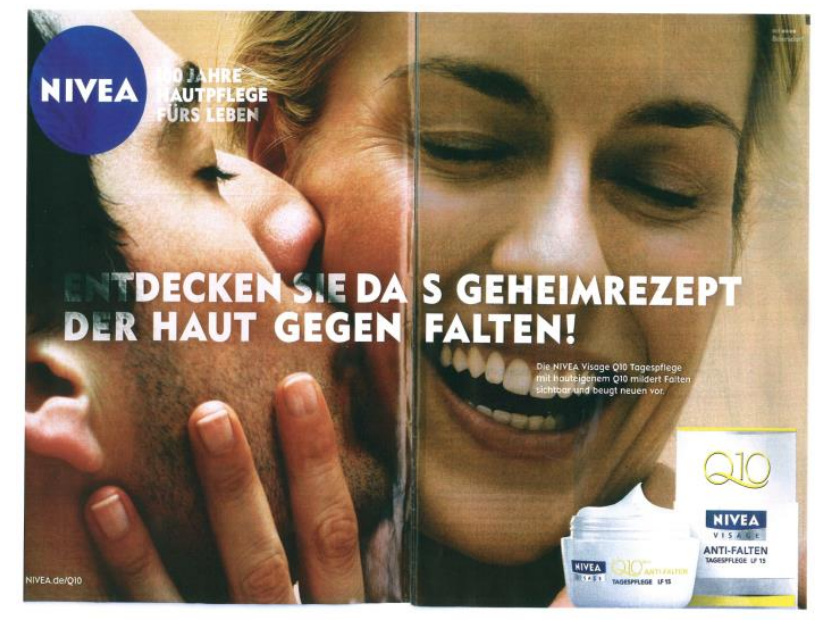

Abbildung 3

Zur sprachlichen Realisierung der Strategie des Problemlösens werden oft auch unvollständige Sätze verwendet. Typisch davon sind Schlagzeilen, die nur aus einem Nebensatz, ohne den Hauptsatz, bestehen. Ein bekanntes Beispiel ist der Slogan von L'Oreal, der in verschiedenen Sprachen realisiert wurde: „Weil Sie es sich wert sind.“ Im Folgenden wird eine Werbung für Augentropfen gezeigt (Abbildung 4), die in der Anzeige einen indirekten Fragesatz ohne den Hauptsatz benutzt: Was ab 50 für Augen wichtig ist. 


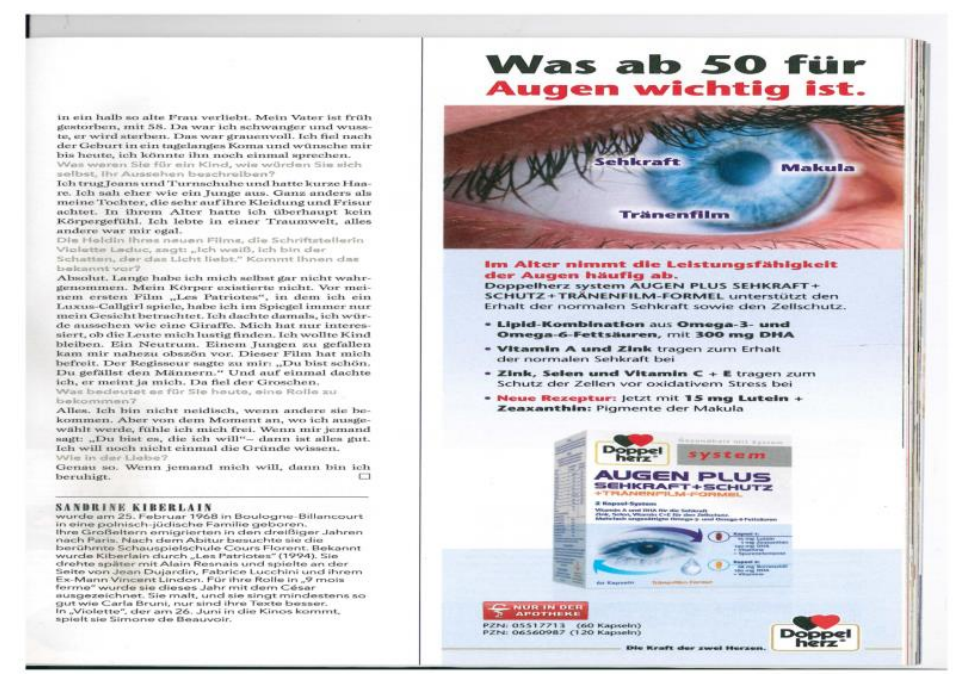

Abbildung 4

Das gleiche Phänomen ist nicht selten in der gesprochenen Sprache zu beobachten, vor allem bei indirekten Fragesätzen, die eine implizite Einladung enthalten („ob wir noch einen Kaffee trinken?"). Außerhalb des kommunikativen Kontexts sind solche Sätze ohne Ambiguität durch die Subjunktion $o b$ zu entschlüsseln. Es handelt sich in diesem Fall um einen Objektsatz ohne Matrixsatz und der passende Hauptsatz kommt in den Gedanken des Hörers dank der Verwendung der Fragepronomen automatisch vor.

Unvollständige Sätze sind in der Werbeliteratur schon lange bekannt. Mit dem Motto „Hauptsachen in Hauptsätzen“ unterstrich Börner (1955: 31) das Bedürfnis, einfache und kurze Propositionen zu benutzen, die typisch für die Massen- und Werbekommunikation sind, damit Empfänger/Empfängerinnen schnell verstehen und dazu ihren passiven Vorstellungsablauf verstärken, ohne große Energie für das Überlegen der Bedeutung aufzuwenden (cf. Ogylvy 1963: 139). Unter unvollständigen Sätzen verstehen die meisten Werbewissenschaftler/Wissenschaftlerinnen elliptische Äußerungen, in denen das Subjekt oder öfter das Prädikat fehlt, oder die nur aus Attributen bestehen, wie:

Männer wie wir - für perfekte Waschergebnisse. AEG Waschgeräte - millionenfach bewährt.

Nivea: satinweiches Hautgefühl - kurz schnell gut.

(Janich 2008: 70; Bußmann 2002: 187)

Im Laufe der Jahre wurde die elliptische Konstruktion zum interphrasalen Bereich ausgedehnt: nicht nur ein Element oder mehrere Elemente eines Satzes werden weggelassen, sondern ein ganzer Satz, nämlich der Hauptsatz, fehlt. Der Nebensatz wird dann wie ein Hauptsatz behandelt und graphisch in Punkte eingeschlossen, mit großem Anfangsbuchstaben geschrieben.

Dieser als Hauptsatz verwendete Nebensatz erschien in meinem Korpus in 11 Anzeigen, und zwar als Kausalsatz (4), Finalsatz (6) sowie Konzessivsatz (1). Einige Beispiele davon sind:

Kausalsätze:

- Weil Sie es sich wert sind (Kosmetika L'Oreal).

- Weil ich im Flugzeug im Zug gesessen habe (Kräuterlikör Jägermeister).

Finalsätze: 
- Um sich in seiner Haut wohlzufühlen (Kosmetika Nivea)

- Damit Sie fliegen, wie Sie es lieben (Fluggesellschaft Condor)

Konzessivsätze:

- Obwohl deine Freunde dir ja nicht folgen (Deo Axe)

Der Hauptsatz wird hier nicht ausgedrückt und gilt deshalb als fakultativ. Es handelt sich um einen expliziten Verzicht auf eine geregelte Sprachform, die Römer unter die Bezeichnung „unvollständige Sätze“ subsumiert hat: Wortfolgen, Wortreihungen, Ellipsen können die Hauptaufmerksamkeit des Empfängers/der Empfängerin auf Begriffe lenken, die gerade durch die Auslassung einiger Satzteile (zum Beispiel der Kopula) wirksam sind (cf. Römer 2002: 167). Ich interpretiere dieses Phänomen in der Werbung als Zeichen für eine potentiell unendliche Assoziationsmöglichkeit seitens der Empfänger/Empfängerinnen, die frei sind, den Text mit einem selbstkonzipierten Hauptsatz zu vervollständigen. Die freie Ergänzungsmöglichkeit und das unendliche Assoziationspotential kennzeichnen Werbetexte durch Äußerungen der Vagheit wie der Mehrdeutigkeit, aber auch durch Teilsätze, die ,einem blank, einer Lücke entsprechen, die potentiell mit allen individuellen Wünschen, Bedürfnissen, Vorstellungen und Erwartungen der Lesenden gefüllt werden kann, um ihnen alles Mögliche zur eigenen Emotionalisierung zu suggerieren" (Ricci Garotti 2016: 90). Daneben erlaubt die argumentative Vagheit der Werbebotschaft, ein breites, unbestimmtes Publikum zu erreichen.

Das Phänomen der Vagheit steht mit der syntaktischen und lexikalischen Genauigkeit, die besonders in wissenschaftlichen Texten zu finden ist, diametral gegenüber; häufig kommt es in Sachbüchern vor, d. h. in Büchern, die Sachthemen und keine Fiktion behandeln, sich aber nicht an Spezialisten richten. Solche Bücher sind praktische Ratgeber für verschiedene Aktivitäten wie Diät oder Sport. In einem Buch zum Radfahren (Brumme 2014) werden zum Beispiel die einzelnen Kapitel durch Nebensätze ohne entsprechenden Hauptsatz übertitelt: „weil man auf dem Rad das Glück kennt"; ,weil man vom Wind etwas lernen kann“; „weil man auch im Wald keine Angst mehr hat". Die gleiche Strategie wird für Artikel in Zeitschriften verwendet, die eine Diät präsentieren oder Ratschläge für die Ernährung anbieten: „Um dich besser zu fühlen“, „um länger und gesünder zu leben“ usw. Wie in kommerziellen Werbetexten wird auch in diesen Texten von einem Problem der Lesenden ausgegangen, für das die Autoren eine Lösung vorschlagen. Der Werbetext bzw. die Werbeproduzenten setzen sich bei dieser Strategie an die Stelle der Empfänger/Empfängerinnen und thematisieren einen kritischen Punkt ihres Lebens, als ob sie nicht in der Lage wären, die eigenen Probleme ohne den Beitrag der Werbekommunikatoren zu erkennen und zu lösen. Die Aufmerksamkeit der Empfänger/Empfängerinnen fokussiert sich somit auf den Effekt der Kaufaktion, nämlich die Lösung, also weder auf die Kaufaktion an sich noch auf den zu kaufenden Artikel. Dies ermöglicht sowohl die Vermeidung einer ausführlichen Beschreibung des Produkts, die Langeweile und Desinteresse erwecken könnte, als auch das Verbergen der persuasiven Absicht, weil die Werbeproduzenten nicht als Verkäufer, sondern als Therapeuten bzw. Heiler und Unterstützer handeln. Infolgedessen wird die positive Auswirkung der Werbebotschaft bekräftigt. 


\section{Themawechsel}

Unter dem Begriff Themawechsel versteht man in der Gesprächsanalyse die Absicht, nicht auf das Thema des Gesprächspartners einzugehen, sondern auf andere Aspekte abzulenken oder den Fortgang des Gesprächs zu modifizieren (cf. Altmann 1981: 48; Thimm 1990: 167). Es handelt sich in diesem Fall um keine kooperative Kommunikation, sogar um eine gezielte Sabotage, in der sowohl die Erwartungen des Gesprächspartners als auch die Maxime der Relation verletzt werden (Marti 2001: 14). Themawechsel ist auch ein Forschungsgegenstand der Textlinguistik, die sich mit Kohärenz und Kohäsion schriftlicher Texte befasst cf. (Hoffmann 1997, Lötscher 1987). Im Werbetext bzw. in der Werbeanzeige hat das Wechseln eines Themas auch das Ziel, die Aufmerksamkeit von Empfängern/Empfängerinnen vom Produkt, also vom Hauptthema, abzulenken. Normalerweise wird der Themawechsel in schriftlichen Texten durch Äußerungen eingeführt, die das Vorhergehende im Text aufgreifen, um auf sekundäre Aspekte einzugehen, damit der Wunsch der Lesenden, das Textthema weiter zu entwickeln, unerfüllt bleibt. Die Aufforderung zum Themawechsel im Werbetext kann sowohl durch textexterne als auch textinterne Objekte erfolgen. In folgenden zwei Beispielen gehe ich auf beide Strategien ein: Im ersten Fall wird das Thema durch einen textexternen, kulturgeprägten Bezug gewechselt. Im zweiten Beispiel wird der Themawechsel mittels textinterner Zeichen, nämlich Konnektoren, herbeigeführt.

\subsection{Themawechsel durch textexterne Bezüge}

Formulierungen von semantisch universellen Allaussagen, die einen allgemeinen Gültigkeitsanspruch vermitteln, werden oft in der Werbung verwendet. Nicht selten handelt es sich um pragmatisch universelle Heterostereotype bzw. Autostereotype, die eine allgemein bekannte Bedeutung sowie eine starke Kanonisierung des Verhaltens voraussetzen. Universell im Sinne von allgemein bekannt sind auf der Sprachebene alle formelhaften Ausdrücke (Klischees, Slogans, Sprichwörter). So wie die Stereotypen können sie einen universell gültigen Sachverhalt explizieren und auch als Aufforderung zum Themawechsel eingesetzt werden. In der Werbung haben Stereotype eine identitätsstiftende Funktion, sie schaffen ein „Wir-Gefühl“", das in der Massenkommunikation immer eine Trennungsfunktion der Angesprochenen von den Anderen, den Nicht-Gemeinten, hat (cf. Ehlich 1989: 21). Die gleiche Trennung zwischen den „Guten“, die das Produkt kaufen bzw. gekauft haben, und den „Miesen“, die nicht angesprochen werden, war lange auch ein implizites Ziel der Werbekommunikation (cf. Ricci Garotti 2004: 29), das derzeit immer noch durch die Benutzung von Stereotypen verstärkt wird. Ein Beispiel für den Themawechsel in der Werbung stellt die Abbildung 5 dar. 


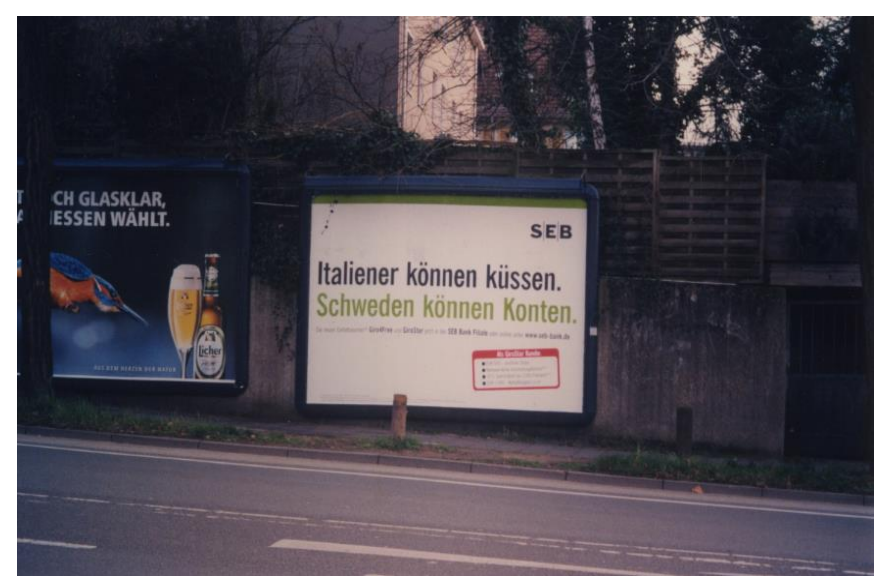

Abbildung 5

\subsection{Themawechsel durch Partikel}

Im Deutschen sind Modalpartikel sowohl als themaindizierende (nun, eigentlich; cf. Brinker/Antos/Hausemann/Sager 2000: 418), als auch als themawechselnde Wörter (ja, doch, eigentlich, cf. Weinrich 1993: 853) klassifiziert worden. Mit Fokuspartikeln werden hingegen bestimmte Aspekte des Gesagten und des Geschriebenen hervorgehoben. Im Folgenden werden zwei Beispiele gezeigt (Abbildung 6 und 7), in denen eine Fokus- und eine Modalpartikel als Zeichen für das Wechseln des Themas verwendet werden.

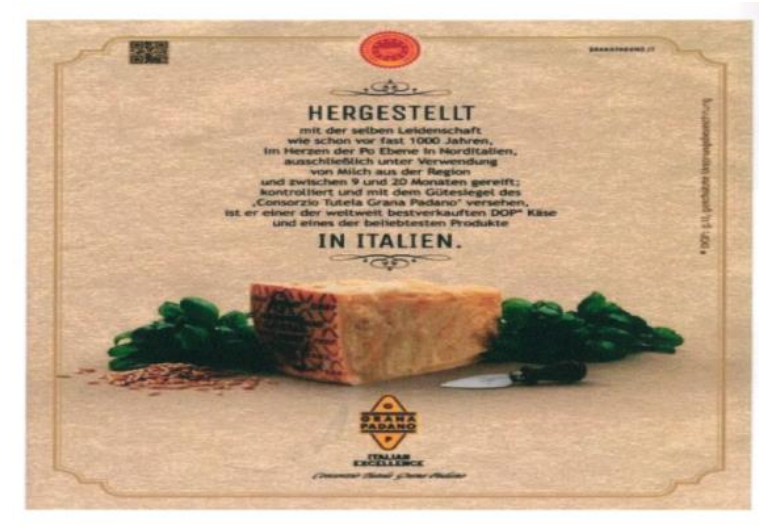

Abbildung 6

Der Satz des Fließtexts lautet: „Hergestellt [groß, markiert] mit derselben Leidenschaft wie schon vor 1000 Jahren“. Die Äußerung wie schon führt einen Partizipialsatz mittels der Kombination wie + schon ein, die oft in Texten aller Sorten in elliptischer und vollständiger Form erscheint, um die Rückkehr zu einem vorhergehenden Thema zu markieren. Werbeproduzenten greifen somit Vorhergehendes auf, indem sie das Wort schon als Fokuspartikel mit temporaler Bedeutung benutzen, so wie in folgenden Beispielen: „wie schon angedeutet", „wie schon erwähnt", ,wie schon gesagt", ,wie schon deutlich wurde“.

Die Äußerung deutet hier auf ein allgemein bekanntes Wissen hin, das aber im Text vorher nie vorkam. Daher wechselt das Thema, das nun nicht mehr das beworbene Produkt ist (der Käse), sondern die italienische Tradition und ihr langzeitiger Erfolg. Verbales und nicht verbales Zeichen stehen sich im Text widersprüchlich gegenüber: Das Bild fokussiert auf das Produkt mittels der key-visual Technik (auch Schlüsselbild, cf. Janich 2003: 62), aber die zwei am Anfang und Ende des Fließtexts markierten Wörter (Hergestellt in Italien) weisen 
auf die italienische Produktion als Garant für Qualität dank der Kompetenz der Produzenten, was scheinbar allgemein bekannt ist. Auf der pragmatischen Ebene handelt es sich um eine Versicherungshandlung, mittels der die Empfänger/Empfängerinnen versichert werden, dass das Beworbene in einer langjährigen Tradition und Kompetenz steht. Verbal wird aber nicht das bildhaft repräsentierte Produkt beschrieben, sondern auf ein anderes Thema fokussiert, das die Hauptargumentation für die Aufforderung der Kaufentscheidung ist.

Die Modalpartikel eigentlich instruiert den Hörer/die Hörerin über einen anstehenden Themawechsel (Weinrich 1993): „was ich eigentlich sagen wollte...“; „was ist eigentlich durch die Erfindung der Null in der Mathematik verändert worden“.

In der Abbildung 7 verweist die Modalpartikel eigentlich auf ein mindestens in Deutschland allgemeines Wissen (die Polemik des Pferdehackfleisches im Ragout, anstelle von Schweineoder Rindfleisch).

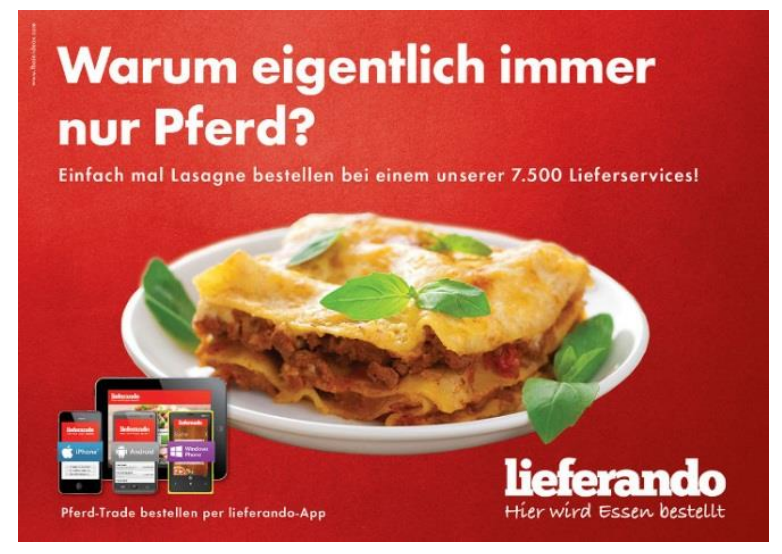

Abbildung 7

Auch in diesem Fall, wie im vorher gezeigten Werbetext über den Grana Padano, wird das Werbeobjekt sowohl im Bild als auch im verbalen Text verborgen: gezeigt wird ein Teller Lasagne und die Schlagzeile thematisiert eben die Zutaten des Gerichts mittels einer rhetorischen, polemischen Frage. Das Werbeobjekt ist hingegen die Lieferfirma lieferando, die nur durch den Markennamen vor dem Slogan gezeigt wird. Das Werbeobjekt wird durch das Hauptbild und die rhetorische Frage verborgen, die vom Produkt durch den Bezug auf das Pferdefleisch mittels der Modalpartikel eigentlich weiter ablenkt. Schlagzeile und Bild stehen nicht nur widersprüchlich einander gegenüber, sondern die eine verschiebt das Thema des zweiten, das das Thema der Werbung (das Produkt) seinerseits verschiebt. Es geht in diesem Fall um einen Meta-Themawechsel, also um eine Kette von Themen, die die Aufmerksamkeit der Empfänger weit über das Anfangsthema hinaus und noch weiter vom Werbeobjekt weg führen.

\section{$4 \quad$ Falsche Popularisierung}

Die dritte Strategie des Verbergens wird in Werbeanzeigen durch die Verwendung von Fachwörtern realisiert. Sie gilt als Zeichen der Fachkompetenz der Werbeproduzenten, die somit als Versicherungshandlung bezüglich ihrer Glaubwürdigkeit einwirkt. Fraglich ist es jedoch, ob die Fachbegriffe zur wissensorientierten Vermittlung in der Werbung beitragen, was eigentlich unter der Bezeichnung von Popularisierung bekannt ist, oder ob es sich nicht viel- 
mehr um eine Strategie handelt, um das Werbeobjekt und die persuasive Handlung durch die prunkvolle Zurschaustellung von Wissenschafts- und Fachsprache darzustellen.

Die Wechselwirkung zwischen Alltags- und Wissenschaftssprache ist lange bekannt, Habermas beschreibt das Phänomen wie folgt:

„Die Umgangssprache hängt freilich von Fach- und Wissenschaftssprache ab. Denn Wissenschaftsfortschritte setzen sich im alltäglichen Bewusstsein dadurch fest, dass Termini (und in seltenen Fällen auch syntaktische Formen) aus einer Wissenschaftssprache in den natürlichen Sprachgebrauch übernommen werden.“

(Habermas 1981: 329).

In der Werbeliteratur wurde die umgekehrte These oft behauptet: Laut Baumgart verstärkt die Umgangssprache die Werbesprache und ist in ihren Bausteinen konzentriert (Schlagzeilen, Slogans, Fließexten usw.; cf. Baumgart 1998: 30). Janich (2001: 4) glaubt hingegen, dass in einigen Werbesektoren eine populärwissenschaftliche Vermittlung möglich ist, die sich besser zur Verbreitung von Fachinhalten und Termini eignet als andere und deren Ziel nicht nur durch persuasive Strategien erreicht wird, sondern auch aufgrund ihrer Informationskraft. Laut Janich kann neues Wissen von der Werbung in die Sprachkultur eintreten und darin konsolidiert werden, nicht zuletzt durch die ständige Wiederholung und Kontextualisierung von neuen Begriffen.

Laut Pörksen (1988: 79) wird der Propagationseffekt bestimmter Wörter und Strukturen der Werbung von ihrer sogenannten „semantischen Plastizität“ garantiert. Angesprochen werden hier die Plastikwörter, also Wörter, in denen die Definitionsmacht absichtlich fehlt, weil man sie gerade aufgrund ihrer Inhaltslosigkeit und Vagheit in unendlich vielen Zusammenhängen benutzen kann. Es handelt sich meistens um entleerte Wörter, die in der Werbung so beliebt sind, dass man sie als Schlüsselwörter fast überall findet, bis sie von den Medien in die Alltagsgespräche übernommen werden. Gerade die Reproduzierbarkeit der Plastikwörter in verschiedenen Kontexten verstärkt ihr Assoziationspotenzial, deshalb gelten sie als ideale Sprachmittel für die Werbegestaltung. ${ }^{1}$

Daher stammt meine Interpretation, dass Fachwörter in der Werbung vor allem eine ästhetische Funktion erfüllen und ein weiteres Mal zum Verbergen des Produkts dienen, statt neue Informationen zu liefern und erklären.

In dem hier untersuchten Korpus kommen Fachwörter relativ oft vor: 9 Anzeigen weisen (oft englische) Termini aus spezifischen Fachbereichen auf, werden aber nur zweimal explizit erklärt: In der Nokia Anzeige erklärt man, was das Wort Flickr heißt, nämlich: ,die weltweit

\footnotetext{
${ }^{1}$ Diese Wörter fehlen in der Wissenschaftssprache völlig, weil sie semantisch leer sind. Amorphe „AmöbenWörter“, wie sie Pörksen genannt hat, sind als Wissenschaftsbegriffe ungeeignet (cf. Pörksen 1988: 17). Wissenschaft braucht genau definierte Termini, um einen Begriff scharf und eindeutig zu fassen.

Trotzdem werden genaue Termini und Definitionen im Werbetext nicht selten missverstanden: Eine im Rahmen der Wiener Dissertationsarbeit „Wortneubildung in der Werbung“ durchgeführte Erhebung hat interessante Interpretationen von Fachtermini in der Werbung gesammelt. Die Vielfalt der von Probanden gelieferten Interpretationen von Fachwörtern in Werbeanzeigen zeigt, dass das Verständnis zum großen Teil aus dem Text und Kontext und weniger aus der eigenen Kompetenz der Empfänger stammt. Auf der anderen Seite kommen Fachwörter sehr häufig in Werbetexten vor, obwohl ihre Bedeutung nicht völlig und eindeutig verstanden wird (cf. Richter 2008).
} 
größte On line Foto-community [...].“ Da es sich um den Namen einer Webseite, also um einen Eigennamen handelt, ist es deshalb fraglich, ob man ihn als Fachterminus bezeichnen kann. Jedenfalls wird es im gleichen Text durch einen zusätzlichen kurzen Exkurs ins Detail erklärt (man kann vermuten, dass die Leser die Bedeutung des Ausdrucks on line community schon kennen). Die zweite Erklärung findet man im Fließtext der Honda Autowerbung, wo ein hinzugefügter Satz näher auf die Bedeutung von LKAS und CMBS als aktiven Spurhalteassistenten und präventivem Fahrerassistenzsystem eingeht.

Manchmal werden Fachtermini benutzt, um ein Thema zu entfalten:

Capital Invest, Ihre führende Fondsgesellschaft mit 50-jähriger Tradition, ist jetzt Pioneer Investments Austria. Pioneer Investments ist seit 1928 höchst erfolgreicher Pionier der Fondsgeschichte. Die Fonds erhalten Sie [...]

(ING DiBa).

Dadurch nehmen automatisch auch die Fachbegriffe zu, wie das folgende Beispiel von Toyota Avensis zeigt:

Der Toyota Avensis. Mit Qualität in jedem Detail: im umfassenden Sicherheitssystemen mit serienmässigem Fahrerknieairbag. Einem Premium Audio System mit MP3/WMA-fähigem CD Player. Dem sprachgesteuerten DVD Navigationssystem

(Toyota)

Im bekannten Beispiel der Activia Werbung, auf die sich die Abbildung 8 bezieht, gelten die dargestellten Bilder vermutlich als Erklärung benutzter Fachtermini.

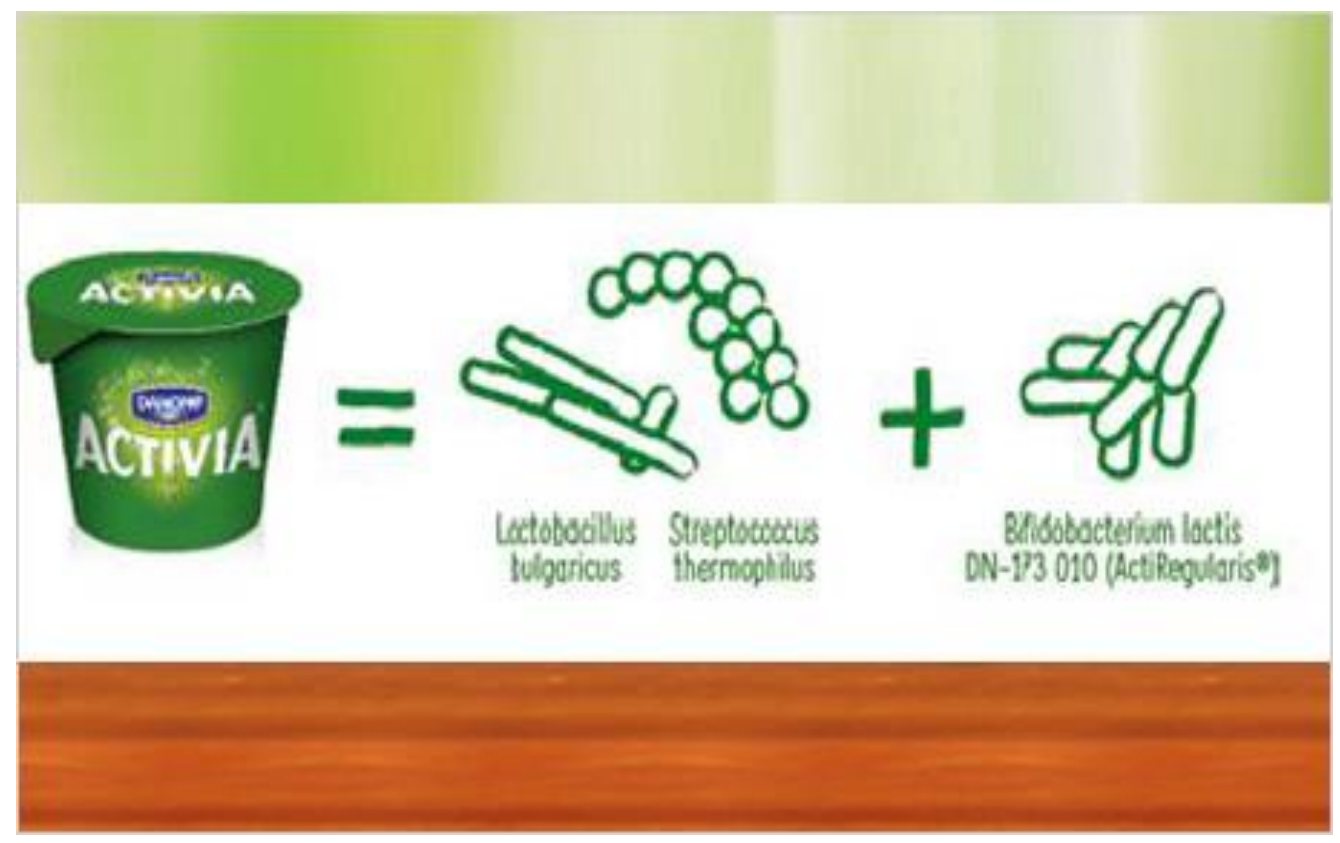

Abbildung 8

Bei näherer Beobachtung weist die Anzeige keine richtige Erklärung, sondern eine einfache Auflistung von Fachtermini auf, die die Lesenden der Fachkompetenz der Hersteller versichern soll. Auch in dem Fließtext vom ähnlichen Produkt Actimel werden Fachtermini nicht erklärt, sondern wirken eher tautologisch ein: 
Neben den ernährungsphysiologischen Eigenschaften, die jedes Milchprodukt ausmachen, beeinflusst Actimel mit seiner besonderen probiotischen Kultur L Casei Defensis bei täglichem Verzehr die körperlichen Abwehrkräfte.

(Actimel)

Man erfährt, dass die probiotische Kultur L Casei Defensis heißt, und man beschreibt den Effekt ihres Gebrauchs (,Abwehrkräfte werden von der Benutzung des Produkts beeinflusst" ${ }^{\text {(") }}$, aber die Ursache des Effekts wird einfach genannt und nicht erklärt. Dass man sie in Milchprodukten findet, kann man aus der ganzen Werbegestaltung schließen, nicht zuletzt aus dem Bild und aus dem Namen des Werbeprodukts; dass sie auf den Körper einen wohltuenden Effekt hat, versteht man einfach aus der Tatsache, dass sie in einer Werbung genannt wird, die quasi per se nur positive Wirkungen bekannt gibt.

Zuletzt ist zu sagen, dass die Benutzung von Fachwörtern in der Werbung kein Indiz einer gewollten Popularisierung, nämlich der Wissensvermittlung durch die werbende Handlung ist. Dazu gelten Fachtermini als persuasive Strategie, die die Aufmerksamkeit der Empfänger durch eine verbal ästhetisierende Operation vom Produkt ablenken. Sie werden als magische Wörter benutzt, deren (meist unbekannte) Bedeutung im Mittelpunkt der Konzentration und der Fokussierung stehen.

Die Analyse unseres Korpus kann zu folgenden Schlussfolgerungen bezüglich der Beziehung zwischen Fach- und Werbesprache führen:

- Fachwörter, die sich auf bestimmte Leistungen der Produkte beziehen, werden im Werbetext nicht erklärt, obwohl gezeigt wurde, dass eine Erklärung notwendig wäre (Richter 2008);

- die Popularisierung ist kein Zweck der Werbung. Fachwörter werden deshalb aus anderen Gründen in die Werbebotschaft eingefügt (Mangel an Zeit und Raum, Notwendigkeit eines Hackstils, Unmittelbarkeit der Botschaft...), die mit dem Hauptziel der Werbung eng verbunden sind (Persuasion, Ablenkung, Überredung);

- Werbung kann allerdings auf Fachtermini nicht verzichten, weil sie ein verstärkendes Element der werbenden Funktion darstellen.

Die Studie von Richter (2008) zur Popularisierungsfunktion der Werbung hat durchaus gezeigt, dass das richtige Verständnis der Fachwörter in der Werbung alles andere als sicher ist, daher gibt es die konkrete Möglichkeit, dass sie auch falsch oder vage in den privaten und öffentlichen Gesprächen von den Empfängern reproduziert werden, die sie nur in Werbetexten gehört/gelesen haben. Versteht man also unter Popularisierung die demokratische und bewusste Vermittlung von Wissenschaftsbegriffen durch populäre Kommunikationskanäle, dann zählt die Werbesprache nicht zu den Kommunikationsformen, die unterschwellig dafür arbeiten.

\section{$5 \quad$ Fazit und Schlusswort}

In einer Diskussion über Sprache und Persuasion kann ein Beitrag zur Werbung nicht fehlen. Es ist durchaus wichtig, in der Werbeforschung nicht von der These auszugehen, dass Werbung lügt, um persuasiv zu sein. Das ist keine Voraussetzung, sondern eine antizipierte Schlussfolgerung, nämlich eine These, die die Werbekommunikation und -kultur als ein iso- 
liertes, nicht zur öffentlichen Kommunikationswelt gehörendes Phänomen betrachtet und auf die Entwicklung einer Sprach-Kommunikationskultivierung von Anfang an verzichtet.

Die Aufgabe der Linguistik und der Kommunikationsforschung ist m. E. so anzusetzen, dass der ganze Kontext der Werbung mit den entsprechenden Forschungsmitteln in allen Elementen, inklusive der Mitwirkung der Empfänger, analysiert wird.

Die Werbeforschung und -literatur ist natürlich auch zeitabhängig: So ergeben sich ständig verändernde Strategien der Werbung mit dem Zweck, an ihr Ziel zu gelangen. Im Beitrag hat man zu zeigen versucht, dass die moderne Werbung weder explizite noch implizite Strategien benutzt, um das Produkt zu verkaufen, sondern in ihrer Gestaltung vorzugsweise eher auf das Produkt selber verzichtet.

Da jede Kommunikationsform Ansporn zur Interpretation des Sozialkontextes geben kann, kann man durch die Analyse der Werbekommunikation eine Vorstellung der sozialen Masseninteraktion in zahlreichen Bereichen bekommen und vermitteln. Schließlich ging es in diesem Beitrag neben der Werbegestaltung und -kommunikation um die Werbesprache. Diesbezüglich möchte ich mit dem Zitat des m. E. besten italienischen Dichters, Giacomo Leopardi, über die Rolle der Sprache in der Gesellschaft schließen, die immer mehr von der Naturwissenschaft und, mit heutigen Worten gesagt, von der Technologie, dominiert wird:

Quindi le lingue guadagnano in precisione, allontanandosi dal primitivo, guadagnano in chiarezza, ordine, regola ecc. Ma in efficacia, varietà ecc. e in tutto ciò ch'è bellezza, perdono sempre quanto più s'allontanano, da quello stato che costituisce la loro primitiva forma

(Giacomo Leopardi 2014: 393)

Die Sprachen gewinnen also an Schärfe, Klarheit, Ordnung, Regelmäßigkeit usw. in dem Maße, wie sie sich von ihrer Ursprünglichkeit entfernen, während sie zugleich an Nachdruck, Buntheit und allem, was schön ist, desto mehr verlieren, je weiter sie von ihrem Naturzustand abkommen.

(Übersetzung ins Deutsche von Hanno Helbling, 1998: 199).

\section{Literaturverzeichnis}

Altmann, Hans (1981): Formen der „Herausstellung “ im Deutschen. Tübingen: Niemeyer. Baumgart, Manuela (1998): Die Sprache der Anzeigenwerbung. Heidelberg: Physika Verlag. Börner, Fritz (1955): Raffiniertes Textschreiben. Aufbau und Formung des Werbetexts. Siegburg. Konstanz/Berlin: Gehlen.

Brinker, Klaus/Antos, Gerd/Heinemann, Wolfgang/Sager, Sven (eds.) (2000): Text- und Gesprächslinguistik. Berlin/New York: de Gruyter.

Brumme, Christoph (2014): 111 Gründe, um Radfahren zu lieben. Berlin: Schwarzkopf \& Schwarzkopf.

Bußmann, Hadumod (2002): Lexikon der Sprachwissenschaft. Stuttgart: Kröner.

Cardinal, Marie (1983): Schattenmund. Die Worte, um es zu sagen. Berlin, Weimar: AufbauVerlag.

Ehlich, Konrad (1989): „Über den Faschismus sprechen. Analyse und Diskurs“. In: Ehlich, Konrad (ed.): Sprache im Faschismus. Frankfurt a. M., Suhrkamp: 7-35.

Habermas, Jürgen (1981): Theorie des kommunikativen Handelns, Vol. I. Frankfurt: Suhrkamp. 
Helbling, Hanno (1998): Giacomo Leopardi. Gesänge, Dialoge und andere Lehrstücke. München: Artemis und Winkler.

Hoffmann, Ludger (1997): „Thematische Organisation von Text und Diskurs“. In: Hoffmann, Ludger, Gisela Zifonun und Bruno Strecker (ed.): Grammatik der deutschen Sprache. Bd. 1. Berlin/New York, de Gruyter: 507-594. (= Schriften des Instituts für deutsche Sprache 7.1).

Knoblauch, Hubert/Raab, Jürgen (2002): „Der Werbespot als kommunikative Gattung“. In: Willems, Herbert (ed.): Die Gesellschaft der Werbung. Wiesbaden, Westdeutscher Verlag: 139-154.

Janich, Nina (1998): Fachliche Informationen und inszenierte Wissenschaft. Tübingen: Narr.

Janich, Nina (2001): „We kehr for you. Werbeslogans und Schlagzeilen als Beitrag zur Sprachkultivierung“. Zeitschrift für angewandte Linguistik 34: 63-81.

Janich, Nina (2003): Werbesprache. Ein Arbeitsbuch. Tübingen: Narr.

Janich, Nina (2008): Textlinguistik: 15 Einführungen. Tübingen: Narr.

Leopardi, Giacomo (2014): Zibaldone, II, edizione I Meridiani, Milano: Mondadori.

Lötscher, Andreas (1987): Text und Thema. Tübingen: Niemeyer.

Marti, Jakob (2001): Verstehensschwierigkeiten bei Nicht-MuttersprachlerInnen. Eine empirische Studie anhand von Aufnahmen bei Asylsuchenden in Bern. Lizentiatsarbeit, Universität Bern. home.datacomm.ch/jakob.marti [21.12.2017]

Ogylvy, David (1963): Confession of an advertising man. London: Atheneum

Pörksen, Uwe (1988): Plastikwörter. Die Sprache einer internationalen Diktatur. Stuttgart: Klett.

Ricci Garotti, Federica (2004): Einführung in die Werbesprache. Trento: Tangram.

Ricci Garotti, Federica (2016): Das Image Italiens in touristischen Reisekatalogen. Roma: Carocci.

Richter, Charlotte (2008): Wortneubildung in der Werbung. Eine kontextorientierte semantische und funktionale Analyse von Wortneubildungen in der Werbesprache. Diplomaarbeit, Universität Wien: germanistik.univie.ac.at/fileadmin/user_upload/inst_germanistik/Wiss_ Arbeiten/Charlotte_Richter.pdf [29.06.2019].

Römer, Ruth (2002): Die Sprache der Anzeigenwerbung. Düsseldorf: Schwann.

Schröder, Jens (2017): Die 50 umsatzstärksten Zeitschriften Deutschlands: Spiegel, stern und Focus trotz starker Verluste vorn meedia.de/2017/03/17/die-50-umsatzstaerksten-zeitschriften-deutschlands-spiegel-sternund-focus-trotz-starker-verluste-vorn [17.3.2017].

Stöckl, Harmut (2008): „Was hat die Werbung zu verstecken?“ In: Pappert, Steffen/Schröter, Melani/Fix, Ulla (eds.): Verschlüsseln, verbergen, verdecken in öffentlicher und institutioneller Kommunikation Berlin, Eric Schmidt: 171-197.

Thimm, Caja (1990): Dominanz und Sprache: Strategisches Handeln im Alltag. Wiesbaden: Springer.

Weinrich, Harald (1993): Textgrammatik der deutschen Sprache. Mannheim: Dudenverlag.

Weinrich, Harald (2000): Linguistik der Lüge. München: Beck.

\section{Anhang}

Gesamte gesammelte Anzeigen 


\begin{tabular}{|l|l|c|}
\hline Produkt & Quelle & Zahl der Anzeigen \\
\hline Ritter & Stern & 2 \\
\hline Frizz-Ease & Stern & 2 \\
\hline Nivea & Stern & 3 \\
\hline Augen plus & Spiegel & 1 \\
\hline Parmigiano & Focus, Stern & 2 \\
\hline Lieferando & Focus, Spiegel & 1 \\
\hline Activia & Stern & 2 \\
\hline L'Oreal & Stern, Spiegel & 3 \\
\hline Jägermeister & Stern & 1 \\
\hline Fluggesellschaft Condor & Spiegel & 3 \\
\hline Nokia & Focus & 4 \\
\hline IngDiBa & Focus & 2 \\
\hline Deo Axe & Stern & 1 \\
\hline Toyota & Spiegel & 3 \\
\hline
\end{tabular}

